\title{
Comparação da Eficácia de Dois Tratamentos Não Farmacológicos para Pacientes com Fibromialgia: Um Estudo Piloto
}

\author{
Comparison of the effectiveness of two non-pharmacological treatments \\ for patients with fibromyalgia: a pilot study
}

\author{
Angélica Cristina Sousa Fonseca ${ }^{1}$, Priscila Conceição Faria ${ }^{1}$, Andrei Pereira \\ Pernambuco ${ }^{1,2,3}$.
}

${ }^{1}$ Centro Universitário de Formiga (UNIFOR-MG) - Faculdade de Fisioterapia. Formiga, Minas Gerais, Brasil.

${ }^{2}$ Universidade Federal de Minas Gerais (UFMG) - Programa de Pós-Graduação em Biologia Celular. Belo Horizonte, Minas Gerais, Brasil.

${ }^{3}$ Universidade de Itaúna (UIT) - Faculdade de Fisioterapia. Itaúna, Minas Gerais, Brasil.

\begin{abstract}
Resumo
Introdução: A fibromialgia (FM) é caracterizada pela presença de dor crônica, generalizada e uma constelação de outros sintomas que se associam a dor. Os fatores que participam de sua gênese e evolução ainda não são completamente compreendidos, o que acaba por dificultar a elaboração de condutas terapêuticas eficazes. Objetivo: Identificar as principais dificuldades encontradas durante a execução de um ensaio clínico randomizado projetado para comparar a eficácia de dois modelos de tratamento não farmacológicos para o tratamento de pacientes com FM. Metodologia: Trinta e quatro mulheres foram inscritas aleatoriamente em um dos dois grupos do estudo, Hidrocinesioterapia $(\mathrm{n}=15)$ ou Escola Inter-Relacional de FM $(n=19)$. Os dois grupos foram submetidos a 11 semanas de intervenção (uma hora por semana). Sete questionários foram utilizados para a coleta dos dados em três momentos distintos. As análises estatísticas foram realizadas no software GraphPad Prism v5.0 com nível de significância de 5\%. Resultados: Apenas 14 pacientes concluíram o estudo, 10 no grupo de hidroterapia e quatro no grupo de educação em saúde. A baixa adesão dos participantes foi a principal dificuldade encontrada na execução do estudo piloto. A Hidrocinesioterapia e a Escola Inter-Relacional de FM surtiram resultados semelhantes em todas as variáveis de interesse: qualidade do sono; níveis de depressão, níveis de ansiedade, descritores da dor, índices de dor, níveis de fadiga, funcionalidade e impacto da FM na qualidade de vida. Conclusão: Os dois modelos de tratamento são equivalentes, entretanto, a baixa adesão dos participantes será o principal desafio a ser sanado em estudos futuros, a fim de torna-los mais consistentes metodologicamente.
\end{abstract}

Palavras Chaves: Fibromialgia. Escola Inter-relacional de Fibromialgia. Hidrocinesioterapia. Tratamento.

Autor correspondente:

Nome: Andrei Pereira Pernambuco

Endereço: Rua Araxá, 206, Morro do Sol.

Itaúna, Minas Gerais - Brasil.

Recebido em: 27/04/2016

Telefone: +553732421477

Revisado em: 06/05/2016

E-mail:pernambucoap@ymail.com

Aceito em: 11/05/2016

Publicado em: 15/06/2016 


\section{Abstract}

Introduction: Fibromyalgia $(F M)$ is characterized by chronic and generalized pain and, a constellation of other symptoms are associated with pain. The factors involved in its genesis and evolution are not fully understood, which makes it difficult to develop effective therapeutic approaches. Objective: To identify the main difficulties encountered during the execution of a randomized clinical trial designed to compare the efficacy of two non-pharmacological treatment models for the treatment of patients with FM. Methodology: Thirty-four women were randomly enrolled in one of two study groups, hydrotherapy $(n=15)$ or Inter-Relational School of FM $(n=19)$. Both groups underwent 11 weeks of intervention (one hour per week). Seven questionnaires were used to collect data at three different times. Statistical analyzes were performed with GraphPad Prism v5.0 software with significance level adjusted to 5\%. Results: Only 14 patients completed the study, 10 in hydrotherapy group and four in health education group. The low adhesion of the participants was the main difficulty encountered in the pilot study execution. The hydrotherapy and the Inter-Relational School of FM have produced similar results in all variables of interest: quality of sleep; levels of depression, anxiety levels, pain descriptors, pain levels, fatigue levels, functionality, and impact of fibromyalgia on the quality of life. Conclusion: The two treatment models are equivalent, however, poor adhesion of the participants will be the main challenge to be resolved in future studies in order to make them more consistent methodologically.

Key Words: Fibromyalgia. Inter-Relational School of Fibromyalgia. Hydrotherapy. Treatment.

\section{Introdução}

A Fibromialgia (FM) é uma síndrome caracterizada pela presença de dor crônica e generalizada. Esta condição acomete cerca de nove mulheres para cada homem acometido. ${ }^{1}$ Os sintomas dolorosos que caracterizam a FM, frequentemente encontram-se associados a outros sintomas como: ansiedade, depressão, intolerância ao exercício, rigidez matinal, fadiga e distúrbios do sono, dentre outros. Apesar dos diversos esforços para elucidar os mecanismos envolvidos na gênese e evolução da FM, tanto a etiologia como a fisiopatologia desta condição ainda são incógnitas para a comunidade médicocientífica. $^{2}$

A complexidade da FM vai além do quadro clínico. Diversas formas de tratamento estão sendo testadas para esta síndrome, contudo, nenhum dos tratamentos utilizados atualmente pode ser considerado completamente eficaz. ${ }^{3}$ A terapêutica farmacológica ainda é a mais utilizada e conta com uma gama de opções como, por exemplo, os antiinflamatórios não esteroides, bloqueadores seletivos de recaptação de serotonina, relaxantes musculares, antidepressivos tricíclicos, dentre outras. ${ }^{4}$ Além do tratamento farmacológico, existem alternativas não farmacológicas, e dentre as opções já testadas, a acupuntura, a yoga, os exercícios aeróbicos, a fisioterapia, a hidroterapia e os programas de educação em saúde (PES) são alternativas que apresentaram um bom custo benefício. ${ }^{5}$

Dentre as diversas técnicas de tratamento por meio da hidroterapia, a Hidrocinesioterapia é a mais utilizada para pacientes com FM. Nesta técnica os exercícios aquáticos são realizados em piscina com água aquecida entre $32^{\circ} \mathrm{C}$ e $33^{\circ} \mathrm{C}$ e os principais objetivos do tratamento são alívio da dor, bem como dos outros sintomas que a acompanham. A redução da dor, se apoia na teoria da comporta da dor de Melzack e Wall, já que durante a imersão, os estímulos sensoriais competem com os estímulos dolorosos, interrompendo assim a transmissão e percepção da dor. ${ }^{6}$ Outros efeitos da Hidrocinesioterapia incluem a diminuição dos espasmos musculares, relaxamento da musculatura, aumento da circulação sanguínea, fortalecimento muscular e melhora da autoestima. ${ }^{7}$

Além da hidroterapia, outros tratamentos não farmacológicos vêm apresentando bons resultados para pacientes com FM e, dentre eles os PES se destacam. ${ }^{8}$ Um bom exemplo de PES específico para o tratamento da FM trata-se da Escola InterRelacional de Fibromialgia (EIF). Este programa foi desenvolvido especificamente para pacientes com FM consiste de uma abordagem multidisciplinar que visa ampliar o conhecimento dos pacientes à respeito de sua condição e das consequências dela resultantes a fim de encorajar os pacientes a buscarem e a atingirem o sucesso terapêutico por meio de seus próprios esforços. ${ }^{9}$

Baseando-se no contexto acima, o objetivo deste estudo piloto foi identificar as principais dificuldades encontradas durante a execução de um ensaio clínico randomizado projetado para comparar a eficácia de dois modelos de tratamentos não farmacológicos para pacientes com FM, a Hidrocinesioterapia e a EIF. 


\section{Metodologia}

\section{Cuidados éticos}

Este estudo foi iniciado após a aprovação do protocolo de pesquisa pelo Comitê de Ética em Pesquisa em Humanos do Centro Universitário de Formiga - MG (CEPH/UNIFOR-MG), todos os procedimentos éticos encontram-se em acordo com a declaração de Helsinque e com a resolução 466/12 do CNS. Para a participação no estudo, todos os voluntários assinaram um Termo de Consentimento Livre e Esclarecido (TCLE) em duas vias.

\section{Tipo de estudo}

Estudo longitudinal, do tipo ensaio clínico randomizado, cego.

\section{Local de realização do estudo}

Todo o processo de amostragem, coleta de dados e intervenção foi realizado em Formiga - MG no campus do UNIFOR - MG (Centro Universitário de Formiga - MG).

\section{Participantes}

Após a busca ativa de voluntárias por meio de anúncios em jornais, e divulgação em Unidades Básicas de Saúde (UBS), a amostragem foi realizada por conveniência. As primeiras 35 voluntárias que se interessaram em participar do estudo e que cumpriram os critérios de inclusão e exclusão foram inscritas no estudo.

\section{Critérios de inclusão e exclusão}

Para participarem do estudo as participantes deveriam possuir diagnóstico de fibromialgia confirmado pelo médico especialista, ter idade entre 25 e 60 anos, IMC abaixo de 30, deveriam assinar o TCLE, e utilizar os mesmos medicamentos há pelo menos três meses (uso estável).

Foram impedidas de participar do estudo aquelas que: não assinaram o TCLE, as que não se encontravam em estabilidade farmacológica de pelo menos três meses, as que possuíam outras enfermidades associadas à fibromialgia, as gestantes e as lactantes.

\section{Aleatorização}

As participantes foram divididas aleatoriamente por sorteio em dois grupos de tratamento: grupo Hidrocinesioterapia (GHIDRO) $(\mathrm{n}=15)$ e grupo Escola Inter-Relacional de FM (GEIF) $(n=19)$.

\section{Instrumentos de coleta e dados}

Sete questionários com validade e confiabilidade asseguradas para a população brasileira foram aplicados por um avaliador experiente que desconhecia os objetivos do estudo, bem como o grupo de tratamento a que pertenciam as participantes. Os questionários utilizados foram: Índice de Qualidade de Sono de Pitsburg; Inventário de Depressão de Beck; Inventário de Ansiedade de Beck; Questionário de Dor de McGill; Escala de
Fadiga de Piper; Medida de Independência Funcional; e Questionário de Impacto da Fibromialgia. Todos os questionários foram aplicados em três momentos distintos: antes do início dos tratamentos, após a sexta semana de intervenção (metade do tratamento) e ao término do tratamento.

\section{Intervenção}

Escola inter-relacional de fibromialgia (EIF)

A EIF consiste de um programa de educação em saúde voltado para pessoas com FM, possui como meta conscientizar e ensinar estratégias de coping e técnicas de auto tratamento aos pacientes com FM. ${ }^{10}$ Esta intervenção tem duração de 11 semanas, com reuniões semanais de 60 minutos cada. Ao total ocorrem nove encontros presenciais com um intervalo de duas semanas (15 dias) entre o sexto e sétimo encontro. ${ }^{9}$

Os temas abordados nas reuniões foram: contrato terapêutico, sintomas, preparação física, preparação mental, respeitar seus limites, nutrição, consequências da cronicidade, tratamento e retrospectiva. E todas as reuniões foram conduzidas por dois pesquisadores devidamente preparados. ${ }^{9}$

\section{$\underline{\text { Hidrocinesioterapia }}$}

Baseado no estudo de Silva et al., (2012), o protocolo de tratamento na Hidrocinesioterapia também teve duração de 11 semanas. A pausa entre a sexta e sétima semana foi implementada a fim de tornar semelhante a frequência das intervenções em ambos os grupos. As sessões de Hidrocinesioterapia também tiveram duração de 60 minutos e foram realizadas uma vez por semana. A intensidade dos exercícios foi moderada (50\% da frequência cardíaca máxima), e os sinais vitais (Preção arterial, pulso e respiração) foram sempre aferidos no inicio e fim dos atendimentos. ${ }^{11}$

O protocolo de atendimento segue quatro fazes: $1^{\mathrm{a}}$ aquecimento global, $2^{\mathrm{a}}$ alongamento muscular, $3^{\mathrm{a}}$ exercícios ativos livres e $4^{\mathrm{a}}$ relaxamento. A água foi mantida à $32^{\circ} \mathrm{C}$ para a realização de todas as atividades. Para o atendimento na piscina foi necessária a presença de dois pesquisadores devidamente treinados, um para conduzir os exercícios hidrocinesioterapêuticos fora da piscina e o outro dentro da piscina. ${ }^{12}$

\section{Análise estatística dos dados}

Para análise dos dados quantitativos (ordinais ou contínuos), primeiramente foi aplicado o teste de Kolmogorov-Smirnov para verificar a normalidade e homogeneidade dos dados. Para avaliação das diferenças entre os grupos (GEIF e GHIDRO) foi utilizado o teste de T de Student Independente (para dados paramétricos) e o teste de Mann-Withney (para dados não paramétricos). Os testes foram realizados no SoftwarePASWStatistics 18 e GraphPad Prism 
V.5.0, aceitando como nível de significância 5\% $(\alpha=0,05)$.

\section{Resultados}

Apenas 14 voluntárias concluíram o estudo piloto, dez no grupo de Hidrocinesioterapia e quatro no grupo da Escola Inter-Relacional de FM.

Os resultados deste estudo piloto sugerem que não existe diferença significativa entre o tratamento com Hidrocinesioterapia em comparação à Escola Inter-relacional de Fibromialgia (EIF) em relação às seguintes variáveis: ansiedade, depressão, MIF, fadiga, FIQ, descritores da dor e índice de dor (TAB. 1). No entanto, é possível observar que antes do tratamento o grupo tratado com Hidrocinesioterapia apresentava níveis de qualidade do sono significativamente piores $(p \leq 0,04)$ do que os do grupo tratado com a EIF, mas ao longo do tratamento essa diferença deixou de existir (TAB 1).

Tabela1: Resultados obtidos após a aplicação dos questionários

$1^{\text {a }}$ Coleta

$2^{\text {a }}$ Coleta

$3^{\text {a }}$ Coleta

\begin{tabular}{|c|c|c|c|c|c|c|}
\hline & $\begin{array}{l}\text { EIF } \\
(n=4)\end{array}$ & $\begin{array}{l}\text { Hidro } \\
(\mathrm{n}=10)\end{array}$ & $\begin{array}{l}\text { EIF } \\
(n=4)\end{array}$ & $\begin{array}{l}\text { Hidro } \\
(\mathrm{n}=10)\end{array}$ & $\begin{array}{l}\text { EIF } \\
(n=4)\end{array}$ & $\begin{array}{l}\text { Hidro } \\
(n=10)\end{array}$ \\
\hline Ansiedade & $30 \pm 8,14$ & $18,5 \pm 11,05$ & $25 \pm 14,15$ & $18 \pm 14,18$ & $24 \pm 9,73$ & $13 \pm 13,36$ \\
\hline Depressão & $16,5 \pm 4,04$ & $18 \pm 6,23$ & $14,5 \pm 3,86$ & $18 \pm 6,48$ & $18,5 \pm 7,96$ & $18 \pm 7,75$ \\
\hline MIF & $121,5 \pm 9,47$ & $121,5 \pm 5,06$ & $124,5 \pm 1,29$ & $125 \pm 4,4$ & $125 \pm 1,16$ & $126 \pm 3,71$ \\
\hline Fadiga & $4,15 \pm 1,82$ & $4,2 \pm 1,91$ & $4,38 \pm 1,35$ & $3,9 \pm 1,95$ & $4,36 \pm 0,92$ & $5,16 \pm 1,66$ \\
\hline FIQ & $66,85 \pm 9,79$ & $62,7 \pm 17,26$ & $59,1 \pm 4,46$ & $55,05 \pm 13,73$ & $55,9 \pm 11,83$ & $52,2 \pm 14,21$ \\
\hline Sono & $7 \pm 3,1^{*}$ & $12,5 \pm 2,87^{*}$ & $11 \pm 3$ & $8,5 \pm 3,8$ & $9 \pm 4,86$ & $8 \pm 1,69$ \\
\hline Descritores dor & $20 \pm 8$ & $20 \pm 4,39$ & $20 \pm 6,69$ & $20 \pm 3,25$ & $20 \pm 4$ & $20 \pm 1,26$ \\
\hline Índice dor & $42,5 \pm 25,51$ & $48 \pm 18,15$ & $40,5 \pm 18,73$ & $50,5 \pm 20,31$ & $53,5 \pm 12,69$ & $53 \pm 13,06$ \\
\hline
\end{tabular}

$*$ Valor de $\mathrm{p} \leq 0,05$

\section{Discussão}

Considerando o caráter crônico da FM e seu impacto negativo na qualidade de vida de seus portadores $^{11}$, torna-se importante a utilização de alternativas não farmacológicas aos tratamentos medicamentosos. Programas de educação em saúde e outras terapias alternativas que tenham sua eficácia comprovada no alívio dos sintomas da FM devem ser incentivadas, já que são soluções de baixo custo, não invasivas e muito abrangentes ${ }^{5}$. Além do mais, as terapias não farmacológicas podem em algumas situações complementar ou até mesmo substituir a terapêutica medicamentosa, que além de não ser completamente eficaz, normalmente se utiliza da associação de diversas classes medicamentosas sem qualquer respaldo da literatura, comprometendo, inclusive, a saúde dos pacientes com FM. ${ }^{3}$

Este estudo piloto foi concluído com 10 mulheres com FM no grupo Hidro e quatro mulheres com FM no grupo EIF. A intenção original era concluir o estudo com o mesmo número de participantes que iniciaram o estudo. Entretanto, logo após o recrutamento das pacientes e a aleatorização, pôde-se observar um alto índice de abandono por parte das voluntárias. A alta taxa de abandono em ambos os grupos, mas principalmente a observada no grupo EIF, pode ter comprometido significativamente os resultados do estudo, visto que em um estudo de Souza et al. (2008), a taxa de adesão foi de $97 \%$, valor muito diferente dos apenas $16 \%$ de adesão verificado no presente estudo. ${ }^{9}$ Evidências apontam como principal dificuldade dos PES a baixa adesão dos pacientes. De acordo com tais evidências, as causas para o abandono do tratamento são diversas e muito particulares. Deste modo, apesar de os PES poderem ocasionar melhoras clínicas, reduzir os gastos públicos e privados decorrentes das doenças, reduzirem a ansiedade do paciente, além de encorajálo a buscar e a atingir o sucesso terapêutico ${ }^{13}$, ainda se fazem necessárias a criação de estratégias que 
minimizem as altas taxas de abandono verificadas neste tipo de programa. ${ }^{14}$ No que se refere ao grupo Hidro, as taxas de abandono foram menores que as identificadas no grupo EIF, mesmo assim também podem ter comprometido de alguma maneira os resultados do estudo. Observa-se assim que o tamanho amostral do estudo foi bastante limitado, o que prejudica a elaboração de conclusões baseadas nos resultados aqui apresentados. Sendo assim, a baixa adesão aos tratamentos propostos deverá ser o principal desafio a ser sanado em estudos futuros que abordarão esta temática. Algumas estratégias precisarão ser pensadas e implementadas a fim de reduzir o abandono por parte dos pacientes. Isto resultaria em uma amostra final mais robusta, o que permitiria a elaboração de conclusões mais precisas. Além disso, a força estatística e a qualidade metodológica do estudo seriam aprimoradas, já que as chances de se incorrer em erro estatístico do tipo II diminuiriam consideravelmente com uma amostragem mais significativa. ${ }^{15}$

Apesar das perdas durante o estudo, pôde ser observado que tanto o tratamento por meio da EIF, como por meio da Hidroterapia apresentaram resultados semelhantes em praticamente todas as variáveis de interesse ou momentos da coleta de dados. Os resultados deste estudo piloto indicam que qualquer uma das intervenções pode ser implementada sem qualquer tipo de desvantagem aos pacientes que receberem ou um ou outro método de tratamento. Ao que parece os dois tratamentos são semelhantes, contudo, o tamanho amostral reduzido prejudica a confirmação desta hipótese. De acordo com algumas evidências, os programas de Hidroterapia para pacientes com FM são capazes de reduzir significativamente o impacto da FM sobre a qualidade de vida dos participantes, melhorar a capacidade funcional, reduzir o absenteísmo ao trabalho, aprimorar a capacidade de serviço, diminuir a intensidade da dor, da fadiga, do cansaço matinal, da rigidez muscular, da ansiedade da depressão, podem ainda melhorar a qualidade do sono e o grau de sonolência diurna. ${ }^{11}$ Todos estes benefícios parecem advir das propriedades físicas da água associadas à rotina de exercícios, esta associação pode estimular a circulação sanguínea e linfática, reduzir o impacto nas articulações e melhorar uma série de sintomas presentes em condições crônicas como a artrite reumatoide e a FM. ${ }^{12}$ Além disso, a Hidrocinesioterapia permite o relaxamento muscular por meio da água aquecida e proporciona aos pacientes fibromiálgicos um tratamento que promove a melhora da autoconfiança, além de favorecer a evolução clínica de forma mais rápida. ${ }^{11}$

Os PES também possuem evidências que apoiam a sua utilização para pacientes com FM. Em estudo pioneiro realizado com a EIF no Brasil, Souza et al. ${ }^{9}$ evidenciaram melhoras significativas no que se refere à gravidade da dor, interferência da dor nas atividades de vida diária, percepção do controle da própria vida, distúrbios emocionais e disposição dos pacientes com FM. Pernambuco et al., em resultados ainda não publicados também evidenciaram que a EIF pode beneficiar os pacientes não só em aspectos subjetivos, mas que podem contribuir para a melhora de aspectos imunológicos e neuroendócrinos, como a concentração de cortisol e de algumas citocinas anti-inflamatórias. Acredita-se que todas estas melhoras possam ser atribuídas ao caráter educativo deste tipo de intervenção, que pode resultar em alterações no comportamento dos envolvidos, estimulando hábitos mais saudáveis e que proporcionem bem estar aos pacientes, já que podem funcionar na prevenção, promoção e recuperação da saúde dos indivíduos. ${ }^{9}$ Além disso, as estratégias de coping podem influenciar diretamente no funcionamento do eixo Hipotálamo-PituitáriaAdrenal (HPA), também conhecido como eixo de resposta ao estresse. Neste sentido, o funcionamento mais ajustado do eixo HPA pode contribuir para o ajuste de importantes biomarcadores da atividade neuroendócrina, tais como: cortisol, adrenalina, noradrenalina, bem como de biomarcadores da atividade imunológica, tais como os níveis de citocinas inflamatórias. ${ }^{16}$

É evidente que o tamanho amostral do presente estudo compromete a confiabilidade dos achados, mas baseado nos achados deste estudo, os dados apontam para uma equivalência entre os dois tipos de tratamento. É importante ressaltar que este estudo trata-se de um piloto e, que será aprimorado a fim de sanar as inconsistências aqui identificadas. Em estudos futuros pretende-se recrutar um maior número de pacientes além de se desenvolver novas estratégias a fim de incentivar os participantes a se manterem em tratamento até o final da $11^{\mathrm{a}}$ semana. Acredita-se que isto permitirá a obtenção de dados mais confiáveis que poderão contribuir com os profissionais de saúde durante o processo de pensamento e tomada de decisão clínica.

\section{Conclusão}

Perante os resultados obtidos, tanto a Hidrocinesioterapia quanto a EIF se equivalem como alternativa não farmacológica para o tratamento da FM. Contudo, insta destacar que os dados aqui apresentados são referentes a um estudo piloto, no qual se encontrou grande dificuldade em manter os participantes ativos durante as 11 semanas de tratamento. Como consequência a amostra final foi pequena e, os achados aqui apresentados precisarão ser confirmados por novos estudos metodologicamente mais consistentes. Mesmo com tais limitações, o estudo cumpriu com seu papel de piloto, afinal os pontos positivos e negativos evidenciados aqui, deverão ser levados em consideração em estudos futuros realizados por este grupo de pesquisa ou por outros pesquisadores interessados nesta temática. 


\section{Declaração de conflitos de interesses}

Os autores do artigo afirmam não haver nenhuma situação de conflito de interesse.

\section{Referências}

1. HAUSER, W.; BURGMER, M.; KOLLNER, V.; SCHAEFERT, R.; EICH, W.; HAUSTEINER-WIEHLE, C.; HENNINGSEN, P. [Fibromyalgia syndrome as a psychosomatic disorder - diagnosis and therapy according to current evidence-based guidelines]. Z Psychosom Med Psychother, v. 59, n. 2, p. 132-52, 2013.

2. MEASE, P.; ARNOLD, L. M.; BENNETT, R.; BOONEN, A.; BUSKILA, D.; CARVILLE, S.; CHAPPELL, A.; CHOY, E.; CLAUW, D.; DADABHOY, D.; GENDREAU, M.; GOLDENBERG, D.; LITTLEJOHN, G.; MARTIN, S.; PERERA, P.; RUSSELL, I. J.; SIMON, L.; SPAETH, M.; WILLIAMS, D.; CROFFORD, L. Fibromyalgia syndrome. J Rheumatol, v. 34, n. 6, p. 1415-25, Jun 2007.

3. BELLATO, E.; MARINI, E.; CASTOLDI, F.; BARBASETTI, N.; MATTEI, L.; BONASIA, D. E.; BLONNA, D. Fibromyalgia syndrome: etiology, pathogenesis, diagnosis, and treatment. Pain Res Treat, v. 2012, p. 426130, 2012.

4. SARZI-PUTTINI, P.; ATZENI, F.; DI FRANCO, M.; BUSKILA, D.; ALCIATI, A.; GIACOMELLI, C.; ROSSI, A.; BAZZICHI, L. Dysfunctional syndromes and fibromyalgia: a 2012 critical digest. Clin Exp Rheumatol, v. 30, n. 6 Suppl 74, p. 143-51, Nov-Dec 2012.

5. BRAZ, A. S.; PAULA, A. P.; DINIZ, M. F. F. M.; ALMEIDA, R. N. Uso da terapia não farmacológica, medicina alternativa e complementar na fibromialgia Rev. Bras. Reumatol., v. vol.51, n. no.3, 2011.

6. BATES, A.; HANSON, N. Síndrome da fibromialgia e exercício aquático. p. 285-99, 1998.

7. SOARES, M. P. Hidroterapia no tratamento da osteoporose. Sprint, 1999.

8. AYAN, C.; ALVAREZ, M. J.; ALONSO-CORTES, B.; BARRIENTOS, M. J.; VALENCIA, M.; MARTIN, V. Health education home-based program in females with fibromyalgia: a pilot study. J Back Musculoskelet Rehabil, v. 22, n. 2, p. 99-105, 2009.

9. SOUZA, J. B.; BOURGAUlT, P.; CHAREST, J.; MARCHAND, S. Escola inter-relacional de fibromialgia: aprendendo a lidar com a dor - estudo clínico randomizado. Rev Bras Reumatol, v. 48, p. 218-225, 2008.

10. GOLD, R. S.; MINER, K. R. Report of the 2000 Joint Committee on Health Education and Promotion Terminology. J Sch Health, v. 72, n. 1, p. 3-7, Jan 2002.

11. SILVA, K. M.; TUCANO, S. J.; KUMPEL, C.; CASTRO, A. A.; PORTO, E. F. Effect of hydrotherapy on quality of life, functional capacity and sleep quality in patients with fibromyalgia. Rev Bras Reumatol, v. 52, n. 6, p. 851-7, Dec 2012

12. PERRATON, L.; MACHOTKA, Z.; KUMAR, S. Components of effective randomized controlled trials of hydrotherapy programs for fibromyalgia syndrome: A systematic review. J Pain Res, v. 2, p. 165-73, 2009.

13. PERNAMBUCO, A. P.; CARVALHO, L. S. C.; REIS, D. A. Impacto de um programa de educação em saúde sobre aspectos neuroimunocomportamentais de pacientes com diagnóstico de fibromialgia. 2014. 126 Ciências Biológicas, Universidade Federal de Minas Gerais

14. LEMSTRA, M.; OLSZYNSKI, W. P. The effectiveness of multidisciplinary rehabilitation in the treatment of fibromyalgia: a randomized controlled trial. Clin J Pain, v. 21, n. 2, p. 166-74, Mar-Apr 2005.

15. MILDNER, G. [A frequent error in statistic computation tables and its elimination]. Z Arztl Fortbild (Jena), v. 61, n. 2, p. 98-105, Jan 151967.

16. PAPADIMITRIOU, A.; PRIFTIS, K. N. Regulation of the hypothalamic-pituitary-adrenal axis. Neuroimmunomodulation, v. 16, n. 5, p. 265-71, 2009. 